6 Cooke RPD, Riordan T, Jones DM, Painter MJ. Secondary cases of meningococcal infection among close family and household contacts in among close family and household contacts in England

7 Olivares R, Hubert B. Clusters of meningococcal disease in France (1987-1988). Eur f Epidemiol 1992;8:737-42.

8 Ege PP, Samuelsson SI, Lind I. [Meningococcal disease 1987-1989. Outbreak of serogroup B:15:PI.16 in the county of Frederiksborg]. Ugeskr Laeger 1993;155:3456-9.

9 Public Health Laboratory Service Communicable Diseases Surveillance Centre Report. $B M \mathcal{Y}$ 1986;293:1293-4.

10 PHLS Meningococcal Infections Working Group and Public Health Medicine Environmental Group. Control of Meningococcal mental Group. Control of Meningococcal disease: guidance for consultants in communicable disease control. Communicable Disease
Report 1995;5(13):R189-93.

\section{Diphtheria: are we ready for it?}

EDrToR,- - The article by Begg and Balraj discusses the adequacy of current control and containment measures for diphtheria. ${ }^{1}$ We agree with the authors that the diagnosis of Corynebacterium diphtheriae and Corynebacterium ulcerans has in the past often been delayed or missed altogether as many laboratories have ceased to culture throat swabs routinely for these organisms.

The Public Health Laboratory Service (PHLS) Standardisation of Clinical Bacteriology Methods Working Group recommends in their standard operating procedure (SOP) on the investigation of throat swabs that all throat swabs should be cultured routinely for $C$ diphtheriae and $C$ ulcerans using Hoyle's tellurite medium. ${ }^{2}$

Reasons for this include:

- Immunisation does not prevent asymptomatic carriage of the organism

- Vaccinated individuals may still be susceptible

- There is a risk of indigenous transmission

- A major outbreak is possible

- Early recognition of a case allows for containment of the patient

- Treatment must be initiated at an early stage to reduce the risk of fatality.

With laboratories returning to this kind of routine screening, isolation of $C$ diphtheriae and $C$ ulcerans from asymptomatic carriers will be increased thereby minimising the potential for missed or delayed diagnosis. It will also allow for the collection of consistent epidemiological data on the presence of the organism in the population.

The PHLS has recently issued a standard method for the investigation of throat swabs as part of the 50 specimen SOPs to be issued to the PHLS during the next 18 months. ${ }^{3}$ These SOPs may also prove useful to microbiology laboratories other than the PHLS.

KURT DJEMAL VAIERIE BEVAN

PHLS, Headquarters Office, 61 Colindale Avenue, London NW9 5DF

1 Begg N, Balraj V. Diphtheria: are we ready for it? Arch Dis Child 1995;73:568-72.

2 Public Health Laboratory Service. PHLS clinical microbiology standard operating procedure for the investigation of throat swabs. B.SOP9; Issue 1. London: PHLS, 1996.

3 Djemal K, Matthews S, Bevan V. Standardisation of clinical bacteriology methods. PHLS Microbiology Digest 1996;13 (1):39-40.
Non-steroidal anti-inflammatory drugs may predispose to invasive group $A$ streptococcal infections

EDrToR,-The suggestion that ibuprofen should be considered as an alternative to paracetamol for the treatment of fever in young children ${ }^{1}$ warrants caution. There have been numerous reports suggesting an association between the use of non-steroidal anti-inflammatory drugs (NSAIDs) and the progression to severe invasive group A streptococcal infections, including necrotising fasciitis. ${ }^{2-6}$ NSAIDs may also mask important clinical features that may help in the early recognition of invasive group A streptococcal disease.

Prompt diagnosis and treatment of group A streptococcal infection has become increasingly important as there has been a worldwide resurgence in invasive group A streptococcal disease since the mid-1980s with the emergence of strains of increased virulence. ${ }^{5}$

Recently, it has been proposed that the underlying biochemical basis for the possible link between the use of NSAIDs and invasive group A streptococcal infection is the ability of NSAIDs to inhibit neutrophil function and enhance cytokine (particularly tumour necrosis factor) production. ${ }^{6}$ In addition, by masking cardinal signs of inflammation, such as myalgia, arthralgia, erythema and localised swelling, these agents may delay the recognition of invasive group A streptococcal infection until signs of shock and multiorgan failure are apparent. This hypothesis may also apply to staphylococcal toxic shock syndrome.

Varicella is an important predisposing factor for both invasive group A streptococcal and staphylococcal infection in immunocompetent children. ${ }^{7}$ NSAIDs may be particularly dangerous in this condition: their use has been associated with the progression to necrotising fasciitis and toxic shock syndrome. ${ }^{4}$

Antipyretics play an important part in the management of febrile young children with non-specific signs in whom the diagnosis is unclear. However, the possibility that NSAIDs may facilitate the invasion of group A streptococci should limit the use of these agents in patients with varicella or in those in whom the cause of fever is not known.

NIGEL CURTIS Paediatric Infectious Diseases Unit, Department of Paediatrics, Imperial College School of Medicine at St Mary's, Queen Elizabeth the Queen Mother Wing, South Wharf Road, London W2 1NY

1 McIntyre J, Hull D. Comparing efficacy and tolerability of ibuprofen and paracetamol in fever. Arch Dis Child 1996;74:164-7.

2 Rimailho A, Riou B, Richard C, Auzepy P. Fulminant necrotizing fasciitis and nonsteroidal anti-inflammatory drugs. $\mathcal{F}$ Infect $D$ is 1987;155: 143-6.

3 Smith RJ, Berk SL. Necrotizing fasciitis and nonsteroidal anti-inflammatory drugs. South Med $\mathcal{F} 1991 ; 84: 785-7$.

4 Brogan TV, Nizet V, Waldhausen JH, Rubens CE, Clarke WR. Group A streptococcal necrotizing fasciitis complicating primary varicella: tizing fasciitis complicating primary varicella: a series of fourteen

5 Curtis N. Invasive group A streptococcal infections. Current Opinion in Infectious Diseases 1996; 9:191-202.

6 Stevens DL. Could nonsteroidal antiinflammatory drugs (NSAIDs) enhance the progression tory drugs (NSAIDs) enhance the progression
of bacterial infections to toxic shock syndrome? of bacterial infections to toxic shoct

7 Pollard AJ, Isaacs A, Lyall EGH, et al. Life threatening bacterial infection associated with varicella. BMF 1996;313:283-5.

\section{HIV related Kaposi's sarcoma}

EDrToR,-McCarthy et al report a Zambian mother and her son with HIV related Kaposi's sarcoma and suggest the possibility of vertical transmission of a Kaposi's sarcoma agent. ${ }^{1}$ They comment on the rarity of Kaposi's sarcoma in the United States and Europe and in their abstract say that it is 'extremely rare in children'.

In Kampala Kaposi's sarcoma is not rare. In a study of children presenting with lymphadenopathy to the paediatric wards of Mulago Hospital, Kampala, between September 1992 and April 1993, 15 cases were diagnosed. The sex ratio was $M: F \quad 10: 3$. Since 1993 about two to three cases are being diagnosed each month. Apart from lymphadenopathy, which can be differentiated from tuberculosis only by histology, other clinical features include pigmented nodular skin lesions, hepatosplenomegaly, pleural effusion, and oral mucosal lesions. We do not have facilities for bronchoscopy, lung puncture, or intestinal biopsy to detect internal lesions. As mothers are not routinely examined in cases of childhood Kaposi's sarcoma we do not know how many might have it. However, it would not be surprising, considering the not uncommon occurrence of Kaposi's sarcoma in women, that both mother and child might have it if they originate from areas of high HIV prevalence such as Uganda or Zambia.

Nevertheless, a transmissible Kaposi's sarcoma agent (whether vertically transmitted or not) is a possibility, and we are also looking for an association with a herpes-like virus.

J B S COULTER
Liverpool School of Tropical Medicine,
Pembroke Place,
Liverpool L3 SQA
C M NDUGWA
Department of Paediatrics and Child Health,
Makerere University,
PO Box 7072, Kampala, Uganda

1 McCarthy GA, Kampmann B, Novelli V, Miller RF, Mercey DE, Gibb D. Vertical transmission of Kaposi's sarcoma. Arch Dis Child of Kaposi's

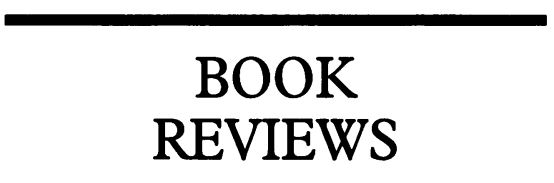

Symptoms of Disease in Childhood. By T J David. (Pp 258; $£ 15.95$ paperback.) Blackwell Science, 1995. ISBN 0-63203635-4.

Updating Common Symptoms of Disease in Children by R S Illingworth, this book follows a symptomatic as opposed to a system approach. For each of the 185 symptom (sign) headings a list of causes is followed by a text, giving a brief account of the conditions listed.

In an attempt to be thorough, many of the lists are lengthy and daunting not only to the medical student but also to the experienced paediatrician. Some attempt has been made to subdivide the causes but further subdivision would have supported the problem solv- 